\title{
Refined enumerations of alternating sign matrices: monotone $(d, m)$-trapezoids with prescribed top and bottom row
}

\author{
Ilse Fischer
}

Received: 13 November 2009 / Accepted: 21 June 2010 / Published online: 17 July 2010

(C) Springer Science+Business Media, LLC 2010

\begin{abstract}
Monotone triangles are plane integer arrays of triangular shape with certain monotonicity conditions along rows and diagonals. Their significance is mainly due to the fact that they correspond to $n \times n$ alternating sign matrices when prescribing $(1,2, \ldots, n)$ as bottom row of the array. We define monotone $(d, m)$-trapezoids as monotone triangles with $m$ rows where the $d-1$ top rows are removed. (These objects are also equivalent to certain partial alternating sign matrices.) It is known that the number of monotone triangles with bottom row $\left(k_{1}, \ldots, k_{n}\right)$ is given by a polynomial $\alpha\left(n ; k_{1}, \ldots, k_{n}\right)$ in the $k_{i}$ 's. The main purpose of this paper is to show that the number of monotone $(d, m)$-trapezoids with prescribed top and bottom row appears as a coefficient in the expansion of a specialisation of $\alpha\left(n ; k_{1}, \ldots, k_{n}\right)$ with respect to a certain polynomial basis. This settles a generalisation of a recent conjecture of Romik et al. (Adv. Math. 222:2004-2035, 2009). Among other things, the result is used to express the number of monotone triangles with bottom row $(1,2, \ldots, i-1, i+1, \ldots, j-1, j+1, \ldots, n)$ (which is, by the standard bijection, also the number of $n \times n$ alternating sign matrices with given top two rows) in terms of the number of $n \times n$ alternating sign matrices with prescribed top and bottom row, and, by a formula of Stroganov for the latter numbers, to provide an explicit formula for the first numbers. (A formula of this type was first derived by Karklinsky and Romik using the relation of alternating sign matrices to the six-vertex model.)
\end{abstract}

Keywords Exact enumeration - Alternating sign matrices $\cdot$ Monotone triangles

Supported by the Austrian Science Foundation FWF, START grant Y463 and NFN grant S9607-N13.

\section{Fischer $(\bowtie)$}

Institut für Mathematik, Universität Klagenfurt, 9020 Klagenfurt, Austria

e-mail: ilse.fischer@univie.ac.at

I. Fischer

Fakultät für Mathematik, Universität Wien, 1090 Wien, Austria 


\section{Introduction}

A monotone triangle is a triangular array of integers of the following form

$$
\begin{aligned}
& \begin{array}{cccc} 
& a_{n, n} & & \\
a_{n-1, n-1} & & a_{n-1, n} & \\
& \ldots & & \ldots
\end{array} \\
& \begin{array}{cccccccccc} 
& & a_{3,3} & & \ldots & & \ldots & \multicolumn{3}{c}{a_{3, n}} \\
\\
a_{1,1} & a_{2,2} & & a_{2,3} & & \ldots & & \ldots & & a_{2, n} \\
& a_{1,2} & & a_{1,3} & & \ldots & & \ldots & & a_{1, n}
\end{array}
\end{aligned}
$$

which is monotone increasing in northeast and in southeast direction and strictly increasing along rows, that is $a_{i, j} \leq a_{i+1, j+1}$ for $1 \leq i \leq j<n, a_{i, j} \leq a_{i-1, j}$ for $1<i \leq j \leq n$ and $a_{i, j}<a_{i, j+1}$ for $1 \leq i \leq j \leq n-1$. Monotone triangles with bottom row $(1,2, \ldots, n)$ are said to be complete and correspond to $n \times n$ alternating sign matrices. The latter are defined as square matrices with entries in $\{0,1,-1\}$ such that in each row and column the sum of entries is 1 and the non-zero entries alternate. Next we display a complete monotone triangle and its corresponding alternating sign matrix.

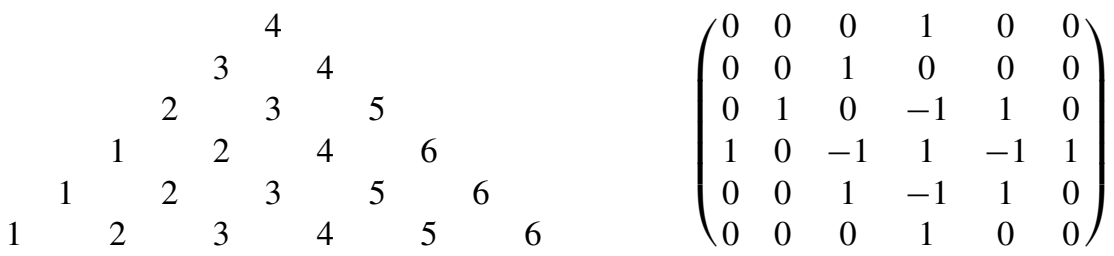

In general, for a given complete monotone triangle $A=\left(a_{i, j}\right)_{1 \leq i \leq j \leq n}$ with $n$ rows, the corresponding $n \times n$ alternating sign matrix $M=\left(m_{i, j}\right)_{1 \leq i, j \leq n}$ can be obtained as follows: we have $m_{i, j}=1$ if and only if

$$
\begin{aligned}
& j \in\left\{a_{n+1-i, n+1-i}, a_{n+1-i, n+2-i}, \ldots, a_{n+1-i, n}\right\} \\
& \backslash\left\{a_{n+2-i, n+2-i}, a_{n+2-i, n+3-i}, \ldots, a_{n+2-i, n}\right\},
\end{aligned}
$$

$m_{i, j}=-1$ if and only if

$$
\begin{array}{r}
j \in\left\{a_{n+2-i, n+2-i}, a_{n+2-i, n+3-i}, \ldots, a_{n+2-i, n}\right\} \\
\backslash\left\{a_{n+1-i, n+1-i}, a_{n+1-i, n+2-i}, \ldots, a_{n+1-i, n}\right\}
\end{array}
$$

and $m_{i, j}=0$ otherwise.

The story of alternating sign matrices [1] started back in the 1980s when Mills, Robbins and Rumsey [7] conjectured that the number of $n \times n$ alternating sign matrices is given by the following beautiful product formula

$$
\prod_{j=0}^{n-1} \frac{(3 j+1) !}{(n+j) !} .
$$


It was more than 10 years later when Zeilberger [9] finally succeeded in giving the first proof of this formula. Since then refined enumerations of alternating sign matrices and enumerations of symmetry classes of alternating sign matrices have been accomplished and fruitful relations to other areas such as statistical physics (six-vertex model) and algebra have been discovered and made the combinatorial analysis of these objects into a major topic in enumerative and algebraic combinatorics as well as in statistical physics.

The central objects of this article are the following generalisations of monotone triangles: for $1 \leq d \leq m$, a monotone $(d, m)$-trapezoid is a monotone triangle with $m$ rows where the first $d-1$ rows are removed. Thus,

$\begin{array}{ccccccccc} & & 2 & & 3 & & 5 & & \\ 1 & 1 & & 2 & & 4 & & 6 & \\ & & 2 & & 3 & & 5 & & 6\end{array}$

is a monotone $(3,5)$-trapezoid. It is easy to translate these objects into the language of alternating sign matrices: for $t \leq n$, let a $(t, n)$-partial alternating sign matrix be a $t \times n$ matrix with entries in $\{0,1,-1\}$ such that the non-zero entries alternate in each row and column and the row-sums are equal to 1 . Suppose $1 \leq i_{1}<$ $i_{2}<\cdots<i_{d} \leq n$ and $1 \leq s_{1}<s_{2}<\cdots<s_{c} \leq n$. Then $(d, n-c)$-trapezoids with top row $i_{1}, \ldots, i_{d}$ and whose bottom row consists of the numbers in $\{1,2, \ldots, n\} \backslash$ $\left\{s_{1}, s_{2}, \ldots, s_{c}\right\}$, arranged in increasing order, are equivalent to $(n-c-d, n)$-partial alternating sign matrices with the following properties: the $j$ th column-sum is 1 iff $j \notin\left\{i_{1}, i_{2}, \ldots, i_{d}, s_{1}, s_{2}, \ldots, s_{c}\right\}$ and it is -1 iff $j \in\left\{i_{1}, \ldots, i_{d}\right\} \cap\left\{s_{1}, \ldots, s_{c}\right\}$. Otherwise, the $j$ th column-sum is 0 and the first non-zero entry (if there is any) of this column is a -1 iff $j \in\left\{i_{1}, \ldots, i_{d}\right\} \backslash\left\{s_{1}, \ldots, s_{c}\right\}$. The $(2,6)$-partial alternating sign matrix that corresponds to the monotone $(3,5)$-trapezoid given above is

$$
\left(\begin{array}{cccccc}
1 & 0 & -1 & 1 & -1 & 1 \\
0 & 0 & 1 & -1 & 1 & 0
\end{array}\right)
$$

Let $\alpha\left(n ; k_{1}, \ldots, k_{n}\right)$ denote the number of monotone triangles with bottom row $\left(k_{1}, \ldots, k_{n}\right)$. In [4] it was shown that

$$
\alpha\left(n ; k_{1}, \ldots, k_{n}\right)=\left(\prod_{1 \leq p<q \leq n}\left(\mathrm{id}+E_{k_{p}} E_{k_{q}}-E_{k_{q}}\right)\right) \prod_{1 \leq i<j \leq n} \frac{k_{j}-k_{i}}{j-i},
$$

where $E_{x}$ denotes the shift operator, defined as $E_{x} p(x)=p(x+1)$. Moreover, note that the product of operators in this formula is understood as the composition. In particular, this operator formula implies that $\alpha\left(n ; k_{1}, \ldots, k_{n}\right)$ is a polynomial in $\left(k_{1}, \ldots, k_{n}\right)$ of degree no greater than $n-1$ in each $k_{i}$. For non-negative integers $c, d$ with $c+d \leq n$, we consider the following expansion:

$$
\alpha\left(n ; k_{1}, \ldots, k_{c}, c+1, c+2, \ldots, n-d, k_{n-d+1}, k_{n-d+2}, \ldots, k_{n}\right)
$$

\footnotetext{
${ }^{1}$ As the monotone $(3,5)$-trapezoid consists of rows 3-5 of our introductory example of a complete monotone triangle, the $(2,6)$-partial alternating sign matrix is the fourth and fifth row of the corresponding alternating sign matrix.
} 


$$
\begin{aligned}
= & \sum_{s_{1}=1}^{n} \sum_{s_{2}=1}^{n} \ldots \sum_{s_{c}=1}^{n} \sum_{i_{1}=1}^{n} \sum_{i_{2}=1}^{n} \ldots \sum_{i_{d}=1}^{n} A\left(n ; s_{1}, s_{2}, \ldots, s_{c} ; i_{1}, \ldots, i_{d}\right) \\
& \times(-1)^{s_{1}+s_{2}+\cdots+s_{c}+c}\left(\begin{array}{c}
k_{1}-c-1 \\
s_{c}-1
\end{array}\right)\left(\begin{array}{c}
k_{2}-c-1 \\
s_{c-1}-1
\end{array}\right) \cdots\left(\begin{array}{c}
k_{c}-c-1 \\
s_{1}-1
\end{array}\right) \\
& \times\left(\begin{array}{c}
k_{n-d+1}-n+d-2+i_{1} \\
i_{1}-1
\end{array}\right) \\
& \times\left(\begin{array}{c}
k_{n-d+2}-n+d-2+i_{2} \\
i_{2}-1
\end{array}\right) \cdots\left(\begin{array}{c}
k_{n}-n+d-2+i_{d} \\
i_{d}-1
\end{array}\right) .
\end{aligned}
$$

The main subject of this paper is the following generalisation of Conjecture 7 from [5], which provides a combinatorial interpretation of the coefficients $A\left(n ; s_{1}\right.$, $s_{2}, \ldots, s_{c} ; i_{1}, \ldots, i_{d}$ ). (In fact, this theorem also implies Conjecture 5 from [5], see Sect. 6.)

Theorem 1 For $1 \leq s_{1}<s_{2}<\cdots<s_{c} \leq n$ and $1 \leq i_{1}<i_{2}<\cdots<i_{d} \leq n$ the coefficient $A\left(n ; s_{1}, \ldots, s_{c} ; i_{1}, \ldots, i_{d}\right)$ is the number of monotone $(d, n-c)$-trapezoids with $\left(i_{1}, \ldots, i_{d}\right)$ as top row and whose bottom row consists of the numbers in $\{1,2, \ldots, n\} \backslash\left\{s_{1}, s_{2}, \ldots, s_{c}\right\}$, arranged in increasing order.

Its proof is the content of Sects. 2-4. In Sect. 5, we use the identity

$$
\alpha\left(n ; k_{1}, \ldots, k_{n}\right)=(-1)^{n-1} \alpha\left(n ; k_{2}, \ldots, k_{n}, k_{1}-n\right)
$$

from [3, Lemma 5] to express the coefficient

$$
A\left(n ; s_{1}, \ldots, s_{c} ; i_{1}, \ldots, i_{d}\right)
$$

in terms of the coefficients

$$
A\left(n ; s_{1}, \ldots, s_{c-t} ; i_{1}, \ldots, i_{d+t}\right),
$$

where $t$ is a fixed non-negative integer no greater than $c$ and $s_{c-l+1} \leq i_{d+l} \leq n$ for $1 \leq l \leq t$. In the special case $c=2, d=0$ and $t=1$ this relates $A\left(n ; s_{1}, s_{2} ;-\right)=$ : $A_{n, s_{1}, s_{2}}$ to $A\left(n ; s_{1} ; i_{1}\right)=: B_{n, s_{1}, i_{1}}$. By the standard bijection between alternating sign matrices and complete monotone triangles, $B_{n, i, j}$ is the number of $n \times n$ alternating sign matrices where the unique 1 in the bottom row is in column $i$ and the unique 1 in the top row is in column $j$. Stroganov [8] has derived a formula for this doubly refined enumeration of alternating sign matrices. On the other hand, the numbers $A_{n, i, j}$ have been studied in [5] and our relation to Stroganov's doubly refined enumeration numbers finally enables us to provide the following formula for the numbers $A_{n, i, j}$ :

$$
\begin{aligned}
A_{n, i, j}= & \frac{1}{A_{n-1}} \sum_{i=1}^{l} \sum_{k=l-i+j}^{l-i+n}(-1)^{n+i+k+l}\left(\begin{array}{c}
2 n-2-j \\
k-l+i-j
\end{array}\right) \\
& \times\left(A_{n-1, l-1}\left(A_{n, k}-A_{n, k-1}\right)+A_{n-1, k-1}\left(A_{n, l}-A_{n, l-1}\right)\right),
\end{aligned}
$$


where

$$
A_{n, k}= \begin{cases}\left(\begin{array}{c}
n+k-2 \\
n-1
\end{array}\right) \frac{(2 n-k-1) !}{(n-k) !} \prod_{j=0}^{n-2} \frac{(3 j+1) !}{(n+j) !} & 1 \leq k \leq n \\
0 & \text { otherwise }\end{cases}
$$

is the number of $n \times n$ alternating sign matrices that have a 1 in the $k$ th column of the first row and $A_{n}$ denotes the total number of $n \times n$ alternating sign matrices. A formula similar to (1.3) was first derived by Karklinsky and Romik [6] by using the six-vertex model approach to alternating sign matrices. Another, complicated, formula for $A_{n, i, j}$ was conjectured in [5, Conjecture 4].)

In fact, the numbers $A_{n, i, j}$ also correspond to a certain doubly refined enumeration of $n \times n$ alternating sign matrices with respect to the two bottom (or, equivalently, two top) rows if $i<j$ : let $k$ be a fixed integer with $i \leq k \leq j$. Then $A_{n, i, j}$ is the number of $n \times n$ alternating sign matrices such that $e_{i}+e_{j}-e_{k}$ is the $(n-1)$ st row and $e_{k}$ is the bottom row where $e_{p} \in \mathbb{R}^{n}$ with $\left(e_{p}\right)_{q}=\delta_{p, q}$. Alternatively and as explained above, it is also the number of $(n-2, n)$-partial alternating sign matrices such that the last non-zero entries (if there exist any) in columns $i$ and $j$ are $-1 \mathrm{~s}$.

\section{How does rotating and reflecting alternating sign matrices translate into the language of complete monotone triangles?}

The set of $n \times n$ alternating sign matrices is invariant under the rotations of $90^{\circ}$ and under the reflection along any of the four symmetry axes of the square. Here, we investigate how these rotations and reflections translate into the language of complete monotone triangles.

Let $A=\left(a_{i, j}\right)_{1 \leq i \leq j \leq n}$ be a complete monotone triangle with $n$ rows and $M$ be the corresponding $n \times n$ alternating sign matrix. We fix the following notation: the sequence $\left(a_{l, l}, a_{l-1, l}, \ldots, a_{1, l}\right)$ is said to be the $l$ th SE-diagonal and the sequence $\left(a_{1, l}, a_{2, l+1}, \ldots, a_{n-l+1, n}\right)$ is said to be the $l$ th NE-diagonal of the complete monotone triangle $A$.

It is not hard to convince oneself that the complete monotone triangle $B=$ $\left(b_{i, j}\right)_{1 \leq i \leq j \leq n}$ which corresponds to the alternating sign matrix that we obtain by reflecting $M$ along the antidiagonal is given by

$$
b_{i, j}=\# \text { of elements } x \text { in the } j \text { th SE-diagonal of } A \text { with } x \geq i .
$$

In other words, one replaces each SE-diagonal of $A$, viewed as a list of parts in an integer partition, by its conjugate partition. We set $B=\mathrm{AD}(A)$. For the example in the introduction we obtain the following monotone triangle and alternating sign matrix.

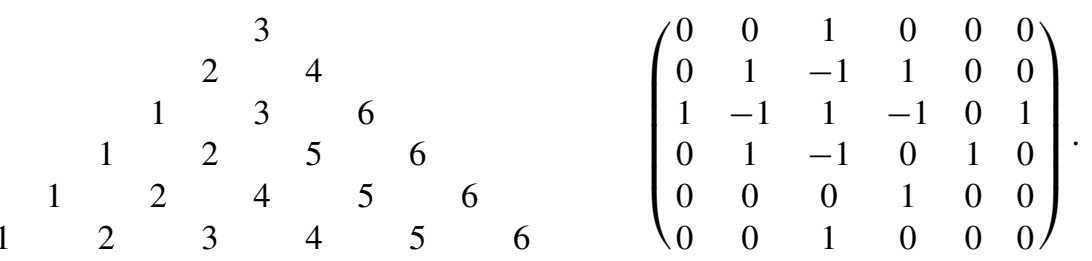


Similarly, the complete monotone triangle $C=\left(c_{i, j}\right)_{1 \leq i \leq j \leq n}$ which corresponds to the alternating sign matrix that we obtain by rotating $M$ clockwise by $90^{\circ}$ is given by

$$
c_{i, j}=\# \text { of elements } x \text { in the }(n+1-j) \text { th NE-diagonal of } A \text { with } x \leq n+1-i \text {. }
$$

We set $C=\operatorname{ROT}(A)$. In the example this gives the following two objects:

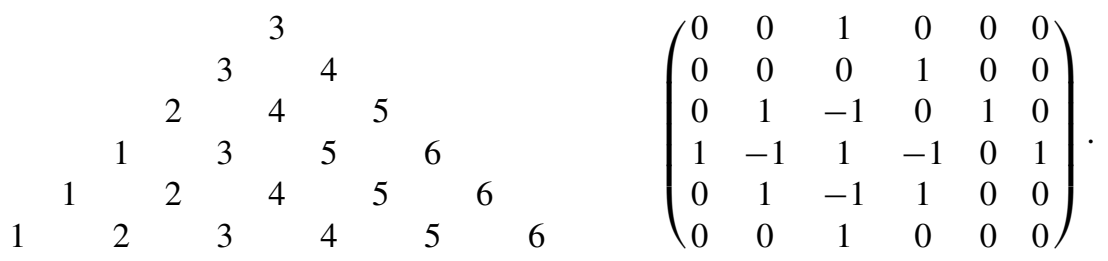

Finally, the complete monotone triangle $D=\left(d_{i, j}\right)_{1 \leq i \leq j \leq n}$ that corresponds to the alternating sign matrix that we obtain by reflecting $M$ along the horizontal symmetry axis is uniquely determined by

$$
\left\{a_{i, i}, a_{i, i+1}, \ldots, a_{i, n}, d_{n+2-i, n+2-i}, d_{n+2-i, n+3-i}, \ldots, d_{n+2-i, n}\right\}=\{1,2, \ldots, n\}
$$

for all $i \in\{1,2, \ldots, n\}$. Here we set $D=\mathrm{H}(A)$. In this case the running example changes to

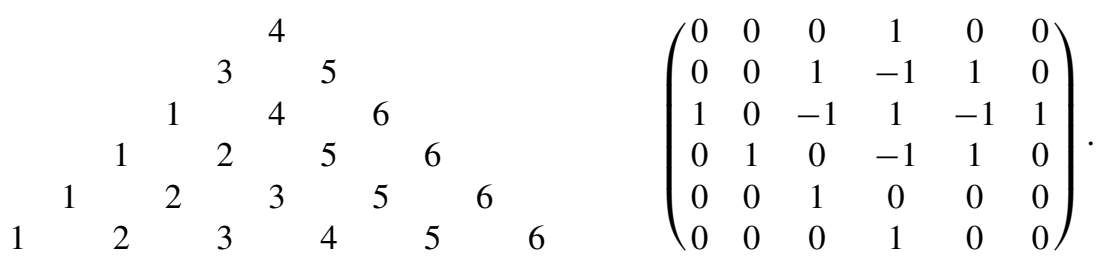

Since reflecting an alternating sign matrix along the antidiagonal is equivalent to first rotating it clockwise by $90^{\circ}$ and then reflecting it along the horizontal symmetry axis we have

$$
\mathrm{AD}=\mathrm{H} \circ \mathrm{ROT} .
$$

However, in the following we do not need the interpretations of the mappings $\mathrm{AD}, \mathrm{ROT}$ and $\mathrm{H}$ in terms of alternating sign matrices. For our purpose, it suffices to show that these three mappings are permutations of the set of complete monotone triangles with $n$ rows (this is easy and left to the reader) and that they satisfy (2.1). The latter is equivalent to showing that for every complete monotone triangle $A=$ $\left(a_{i, j}\right)_{1 \leq i \leq j \leq n}$ and $i \in\{1,2, \ldots, n\}$ the union of

$$
\{\# \text { of elements } x \text { in } j \text { th SE-diagonal of } A \text { with } x \geq i \mid i \leq j \leq n\}
$$

and

$$
\{\# \text { of elements } x \text { in the } j \text { th NE-diagonal of } A \text { with } x \leq i-1 \mid 1 \leq j \leq i-1\}
$$


Fig. $1 p_{1}=5, p_{2}=4, p_{3}=2$, $p_{4}=1, q_{5}=3, q_{6}=6$
○

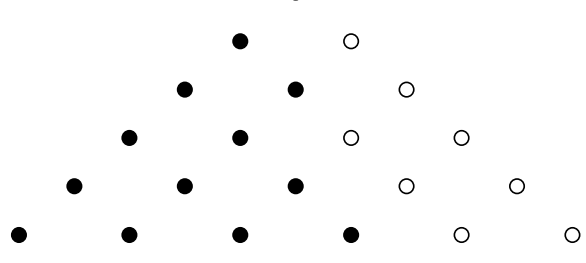

is equal to $\{1,2, \ldots, n\}$. This follows from the following fact, see also Fig. 1: suppose $\left(p_{1}, p_{2}, \ldots, p_{i-1}\right)$ is a strictly decreasing sequence of positive integers no greater than $n$ and for $1 \leq j \leq i-1$ delete the first $p_{j}$ entries from the $j$ th NE-diagonal of a triangular array of dots with $n$ rows that corresponds to the shape of a monotone triangle with $n$ rows. For $i \leq j \leq n$, let $q_{j}$ be the length of the $j$ th SE-diagonal in the remaining array of dots. Then

$$
\left\{p_{1}, \ldots, p_{i-1}, q_{i}, q_{i+1}, \ldots, q_{n}\right\}=\{1,2, \ldots, n\} .
$$

Indeed, if $k$ is any number between 1 and $n$, and $k$ does not appear in the list $p_{1}, p_{2}, \ldots, p_{i-1}$, then $k$ must be between $p_{j}$ and $p_{j+1}$ (in the strict sense) for some $j$ (we set $p_{0}=n+1$ and $p_{i}=0$ ). The assertion now follows as, for $j=0,1, \ldots, i-1$, we have $q_{j+k}=k$ for $k \in\left\{p_{j+1}+1, p_{j+1}+2, \ldots, p_{j}-1\right\}$. (However, the assertion also follows easily by induction with respect to $n$ as either $p_{1}=n$ or $q_{n}=n$.)

\section{From monotone $(d, n-c)$-trapezoids with prescribed top and bottom row to monotone arrays}

In this section we fix the two sequences $\left(i_{1}, \ldots, i_{d}\right)$ and $\left(s_{1}, \ldots, s_{c}\right)$ with $1 \leq i_{1}<$ $i_{2}<\cdots<i_{d} \leq n$ and $1 \leq s_{1}<s_{2}<\cdots<s_{c} \leq n$. We define monotone arrays of size $n$ that are associated with these sequences as follows: the shape of these integer arrays is that of a monotone triangle with $n$ rows where for $1 \leq l \leq c$ the first $s_{c+1-l}$ entries of the $l$ th NE-diagonal are missing and for $n-d+1 \leq l \leq n$ the last $i_{l-n+d}$ entries of the $l$ th SE-diagonal are missing. The entries of the monotone array are again weakly increasing along the truncated NE-diagonals as well as along the SE-diagonals and strictly increasing along rows.

We will use the mappings from the previous section to show that the number of monotone $(d, n-c)$-trapezoids with $\left(i_{1}, \ldots, i_{d}\right)$ as top row and whose bottom row consists of the numbers in $\{1,2, \ldots, n\} \backslash\left\{s_{1}, s_{2}, \ldots, s_{c}\right\}$, arranged in increasing order, is equal to the number of these monotone arrays with entries in $\{c+1, c+2, \ldots, n-d\}$.

Indeed, fix such a monotone $(d, n-c)$-trapezoid and add entries arbitrarily to make it up to a complete monotone triangle $A$ with $n$ rows. As an example, we take the $(3,5)$-trapezoid on the left and the completion on the right, i.e. $c=1$ and $n=6$. 


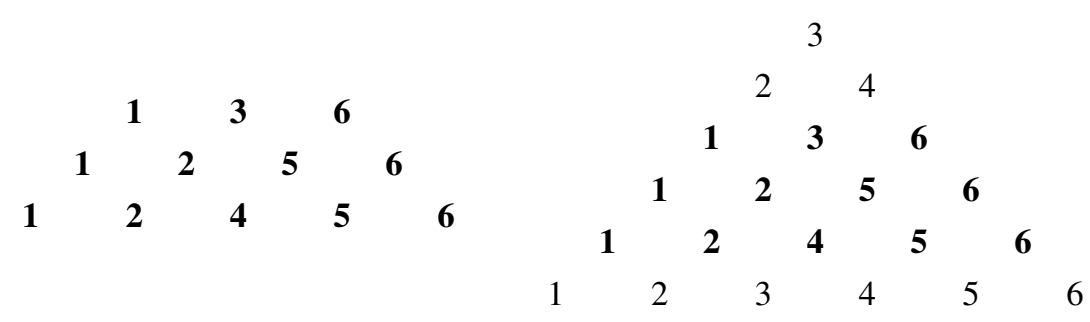

Consider $B=\mathrm{AD}^{-1}(A)$. In our example $B$ is the following complete monotone triangle:

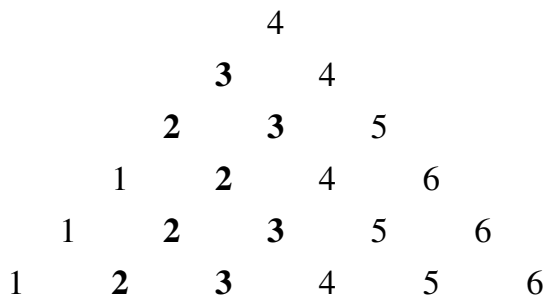

(The entries $c+1, c+2, \ldots, n-d$ are displayed in boldface as they correspond to the information contained in the original monotone $(3,5)$-trapezoid.) By the definition of $\mathrm{AD}$, the elements greater than $n-d$ in $B$ are just the last $i_{l-n+d}$ elements in the $l$ th SE-diagonals where $n-d+1 \leq l \leq n$ and the distribution of these entries is exactly determined by the completion of the monotone $(d, n-c)$-trapezoid towards the top. On the other hand, by (2.1), $B=\mathrm{ROT}^{-1} \circ \mathrm{H}^{-1}(A)$. Thus, the $c$ th row of $\mathrm{H}^{-1}(A)$ (counted from the top) is $\left(s_{1}, \ldots, s_{c}\right)$ and the first $c-1$ rows of this complete monotone triangle correspond to the completion of the original monotone $(d, n-c)$-trapezoid towards the bottom. Consequently (and by the definition of ROT), the elements no greater than $c$ of $B$ are just the first $s_{c+1-l}$ elements in the $l$ th NE-diagonals for $1 \leq l \leq c$ and the distribution of these entries is determined by the completion of the monotone $(d, n-c)$-trapezoid towards the bottom.

If we delete in our example the first $s_{c+1-l}$ entries in the $l$ th NE-diagonal for $1 \leq l \leq c$ and the last $i_{l-n+d}$ elements in the $l$ th SE-diagonal for $n-d+1 \leq l \leq$ $n$ (i.e. exactly the part of the complete monotone triangles that corresponds to the completion of the original monotone $(d, n-c)$-trapezoid towards top and bottom), we clearly obtain the following monotone array.

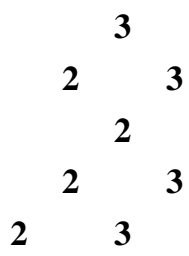

For technical reasons (which will become apparent later) we add the entry $c+1$ at the beginning of the truncated $l$ th NE-diagonal for $1 \leq l \leq c$ and add the entry $n-d$ at the end of the truncated $l$ th SE-diagonal for $n-d+1 \leq l \leq n$. In our example, we obtain 


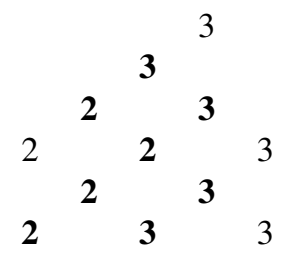

This will certainly not destroy the monotonicity along diagonals, but may disturb the strict increase along rows. All in all we see that the number of monotone $(d, n-c)$ trapezoids with fixed top and bottom row as given above is the number of "extended" monotone arrays with entries in $\{c+1, c+2, \ldots, n-d\}$, where for $1 \leq l \leq c$ the first $s_{c+1-l}-1$ entries of the $l$ th NE-diagonal are missing and the first entry of the truncated diagonal is equal to $c+1$ and does not necessarily have to be strictly smaller than its neighbour to the right and for $n-d+1 \leq l \leq n$ the last $i_{l-n+d}-1$ entries in the $l$ th SE-diagonal are missing and the last entry of the truncated diagonal is equal to $n-d$ and does not necessarily have to be strictly greater than its neighbour to the left.

\section{Completion of the proof of Theorem 7}

Suppose $A\left(l_{1}, \ldots, l_{n-1}\right)$ is a function in $\left(l_{1}, \ldots, l_{n-1}\right)$ and $\left(k_{1}, \ldots, k_{n}\right) \in \mathbb{Z}^{n}$. We define a summation operator

$$
\sum_{\left(l_{1}, \ldots, l_{n-1}\right)}^{\left(k_{1}, \ldots, k_{n}\right)} A\left(l_{1}, \ldots, l_{n-1}\right)
$$

by induction with respect to $n$. If $n=0$ then the application of the operator gives zero, for $n=1$ we set

$$
\sum_{(-)}^{k_{1}} A=A
$$

If $n \geq 2$ then we define

$$
\begin{aligned}
\sum_{\left(l_{1}, \ldots, l_{n-1}\right)}^{\left(k_{1}, \ldots, k_{n}\right)} A\left(l_{1}, \ldots, l_{n-1}\right)= & \sum_{\left(l_{1}, \ldots, l_{n-2}\right)}^{\left(k_{1}, \ldots, k_{n-1}\right)} \sum_{l_{n-1}=k_{n-1}}^{k_{n}} A\left(l_{1}, \ldots, l_{n-1}\right) \\
& -\sum_{\left(l_{1}, \ldots, k_{n-2}\right)}^{\left(l_{1}, \ldots, l_{n-3}\right)} A\left(l_{1}, \ldots, l_{n-3}, k_{n-1}, k_{n-1}\right) .
\end{aligned}
$$

With this, we obviously have

$$
\alpha\left(n ; k_{1}, \ldots, k_{n}\right)=\sum_{\left(l_{1}, \ldots, l_{n-1}\right)}^{\left(k_{1}, \ldots, k_{n}\right)} \alpha\left(n-1 ; l_{1}, \ldots, l_{n-1}\right)
$$


for $n \geq 2$. In fact, if we extend the definition of monotone triangles to triangular integer arrays $\left(a_{i, j}\right)_{1 \leq i \leq j \leq n}$ with weak increase along NE-diagonals and SEdiagonals and strict increase along rows with the possible exception of the bottom row which may only be weakly increasing then $\alpha\left(n ; k_{1}, \ldots, k_{n}\right)$ is also the number of these (extended) monotone triangles with bottom row $\left(k_{1}, \ldots, k_{n}\right)$. Recall that $E_{x} p(x)=p(x+1)$, let $\delta_{x}=\mathrm{id}-E_{x}^{-1}$ and set

$$
B\left(k_{1}, \ldots, k_{n}\right)=\sum_{\left(l_{1}, \ldots, l_{n-1}\right)}^{\left(k_{1}, \ldots, k_{n}\right)} A\left(l_{1}, \ldots, l_{n-1}\right) .
$$

The recursion shows that

$$
\delta_{k_{n}} B\left(k_{1}, \ldots, k_{n}\right)=\sum_{\left(l_{1}, \ldots, l_{n-2}\right)}^{\left(k_{1}, \ldots, k_{n-1}\right)} A\left(l_{1}, \ldots, l_{n-2}, k_{n}\right)
$$

and, more generally for $d \geq 1$,

$$
\delta_{k_{n-d+1}} \delta_{k_{n-d+2}} \ldots \delta_{k_{n}} B\left(k_{1}, \ldots, k_{n}\right)=\sum_{\left(l_{1}, \ldots, l_{n-d-1}\right)}^{\left(k_{1}, \ldots, k_{n-d}\right)} A\left(l_{1}, \ldots, l_{n-d-1}, k_{n-d+1}, \ldots, k_{n}\right) .
$$

Similarly, with $\Delta_{x}=E_{x}-\mathrm{id}$, it is not hard to see that

$$
(-1)^{c} \Delta_{k_{1}} \Delta_{k_{2}} \ldots \Delta_{k_{c}} B\left(k_{1}, \ldots, k_{n}\right)=\sum_{\left(l_{c+1}, \ldots, l_{n-1}\right)}^{\left(k_{c+1}, \ldots, k_{n}\right)} A\left(k_{1}, \ldots, k_{c}, l_{c+1}, \ldots, l_{n-1}\right) .
$$

If we combine these two facts we see that

$$
\begin{aligned}
\Delta_{k_{1}} \Delta_{k_{2}} \ldots \Delta_{k_{c}} \delta_{k_{n-d+1}} \delta_{k_{n-d+2}} \ldots \delta_{k_{n}}(-1)^{c} B\left(k_{1}, \ldots, k_{n}\right) \\
=\sum_{\left(l_{c+1}, \ldots, l_{n-d-1}\right)}^{\left(k_{c+1}, \ldots, k_{n-d}\right)} A\left(k_{1}, \ldots, k_{c}, l_{c+1}, \ldots, l_{n-d-1}, k_{n-d+1}, \ldots, k_{n}\right) .
\end{aligned}
$$

For $1 \leq i_{1} \leq i_{2} \leq \cdots \leq i_{d} \leq n$ and $1 \leq s_{1} \leq s_{2} \leq \cdots \leq s_{c} \leq n$, let

$$
\gamma\left(n ; k_{1}, \ldots, k_{n} ; s_{1}, \ldots, s_{c} ; i_{1}, \ldots, i_{d}\right)
$$

(see Fig. 2) denote the number of extended monotone arrays with $n$ rows that have the following properties.

- For $1 \leq l \leq c$, the first $s_{c+1-l}-1$ entries of the $l$ th NE-diagonal are missing.

- The first entry of the truncated $l$ th NE-diagonal is equal to $k_{l}$ and does neither have to be strictly smaller than its neighbour to the right nor weakly smaller than its SE-neighbour.

- For $n-d+1 \leq l \leq n$, the last $i_{l-n+d}-1$ entries in the $l$ th SE-diagonal are missing. 


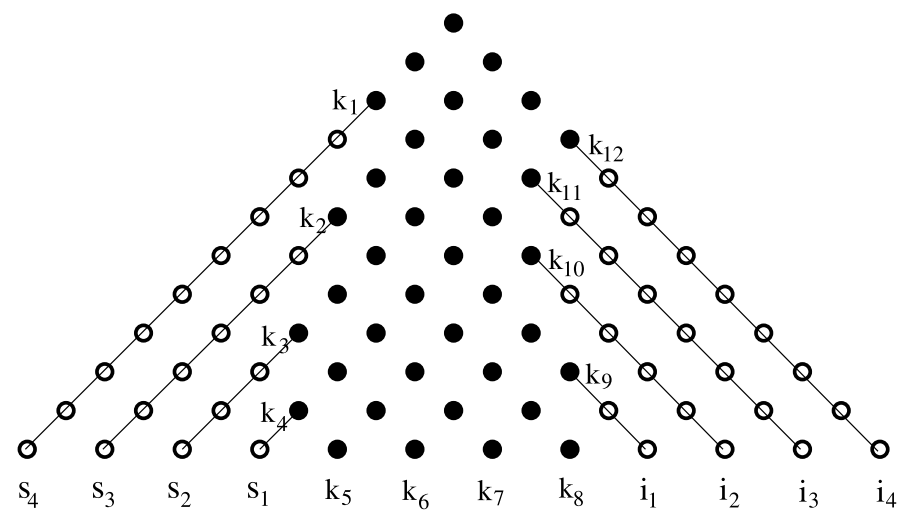

Fig. 2 In this example we have $n=12, c=d=4, s_{1}=2, s_{2}=4, s_{3}=7, s_{4}=10$ and $i_{1}=3, i_{2}=6, i_{3}=8, i_{4}=9$

- The last entry of the truncated $l$ th SE-diagonal is equal to $k_{l}$ and does neither have to be strictly greater than its neighbour to the left nor weakly greater than its SWneighbour.

- The bottom row (without those entries among $k_{1}, k_{2}, \ldots, k_{c}, k_{n-d+1}, k_{n-d+2}$, $\ldots, k_{n}$ that are situated in the bottom row) is equal to $k_{c+1}, k_{c+2}, \ldots, k_{n-d}$ and we do not demand a strict increase for those entries.

We want to show that

$$
\begin{aligned}
& \gamma\left(n ; k_{1}, \ldots, k_{n} ; s_{1}, \ldots, s_{c} ; i_{1}, \ldots, i_{d}\right) \\
& \quad=\Delta_{k_{1}}^{s_{c}-1} \Delta_{k_{2}}^{s_{c-1}-1} \ldots \Delta_{k_{c}}^{s_{1}-1} \delta_{k_{n-d+1}}^{i_{1}-1} \delta_{k_{n-d+2}}^{i_{2}-1} \ldots \delta_{k_{n}}^{i_{d}-1}(-1)^{s_{1}+\cdots+s_{c}-c} \alpha\left(n ; k_{1}, \ldots, k_{n}\right)
\end{aligned}
$$

by induction with respect to $n$. The case $n=1$ is easy to check. Otherwise, let $c^{\prime} \geq 0$ be minimal such that $s_{c^{\prime}+1}>1$ and $d^{\prime} \geq 0$ be minimal such that $i_{d^{\prime}+1}>1$. Then we have the following recursion for $\gamma$ with respect to $n$ :

$$
\begin{aligned}
\gamma\left(n ; k_{1}, \ldots, k_{n} ; s_{1}, \ldots, s_{c} ; i_{1}, \ldots, i_{d}\right) & \\
= & \sum_{\left(l_{c-c^{\prime}+1}, \ldots, l_{n-d+d^{\prime}-1}\right)}^{\left(k_{c-c^{\prime}+1}, \ldots, k_{n-d+d^{\prime}}\right)} \gamma\left(n-1 ; k_{1}, \ldots, k_{c-c^{\prime}}, l_{c-c^{\prime}+1}, \ldots, l_{n-d+d^{\prime}-1}, k_{n-d+d^{\prime}+1}, \ldots, k_{n}\right. \\
& \left.s_{c^{\prime}+1}-1, s_{c^{\prime}+2}-1, \ldots, s_{c}-1 ; i_{d^{\prime}+1}-1, i_{d^{\prime}+2}-1, \ldots, i_{d}-1\right) .
\end{aligned}
$$

By the induction hypothesis, this is equal to

$$
\begin{gathered}
\sum_{\left(l_{c-c^{\prime}+1}, \ldots, l_{n-d+d^{\prime}-1}\right)}^{\left(k_{c-c^{\prime}+1}, \ldots, k_{n-d+d^{\prime}}\right)} \Delta_{k_{1}}^{s_{c}-2} \Delta_{k_{2}}^{s_{c-1}-2} \ldots \Delta_{k_{c-c^{\prime}}}^{s_{c^{\prime}+1}-2} \delta_{k_{n-d+d^{\prime}+1}}^{i_{d^{\prime}+1}-2} \ldots \delta_{k_{n}}^{i_{d}-2}(-1)^{\left(s_{c^{\prime}+1}-1\right)+\cdots+\left(s_{c}-1\right)-c+c^{\prime}} \\
\alpha\left(n-1 ; k_{1}, \ldots, k_{c-c^{\prime}}, l_{c-c^{\prime}+1}, \ldots, l_{n-d+d^{\prime}-1}, k_{n-d+d^{\prime}+1}, \ldots, k_{n}\right) .
\end{gathered}
$$


Now, (4.1) implies that this is furthermore equal to

$$
\begin{aligned}
& \Delta_{k_{1}}^{s_{c}-2} \Delta_{k_{2}}^{s_{c-1}-2} \ldots \Delta_{k_{c-c^{\prime}}}^{s_{c^{\prime}+1}-2} \delta_{k_{n-d+d^{\prime}+1}}^{i_{d^{\prime}+1}-2} \ldots \delta_{k_{n}}^{i_{d}-2}(-1)^{s_{c^{\prime}+1}+\cdots+s_{c}} \\
& \Delta_{k_{1}} \Delta_{k_{2}} \ldots \Delta_{k_{c-c^{\prime}}} \delta_{k_{n-d+d^{\prime}+1}} \delta_{k_{n-d+d^{\prime}+2}} \ldots \delta_{k_{n}}(-1)^{c-c^{\prime}} \alpha\left(n ; k_{1}, \ldots, k_{n}\right) .
\end{aligned}
$$

This concludes the proof of (4.2).

By the observations from the previous section and (4.2), the number of $(d, n-c)$ trapezoids with $\left(i_{1}, \ldots, i_{d}\right)$ as top row and whose bottom row consists of the numbers in $\{1,2, \ldots, n\} \backslash\left\{s_{1}, s_{2}, \ldots, s_{c}\right\}$, arranged in increasing order, is equal to

$$
\begin{aligned}
& \Delta_{k_{1}}^{s_{c}-1} \Delta_{k_{2}}^{s_{c-1}-1} \ldots \Delta_{k_{c}}^{s_{1}-1} \delta_{k_{n-d+1}}^{i_{1}-1} \delta_{k_{n-d+2}}^{i_{2}-1} \ldots \delta_{k_{n}}^{i_{d}-1} \\
& \quad \times\left.(-1)^{s_{1}+\cdots+s_{c}-c} \alpha\left(n ; k_{1}, \ldots, k_{n}\right)\right|_{\left(k_{1}, \ldots, k_{n}\right)=\left((c+1)^{c}, c+1, c+2, \ldots, n-d,(n-d)^{d}\right)},
\end{aligned}
$$

where $(c+1)^{c}$ denotes the sequence of length $c$, which contains only $c+1$, and $(n-d)^{d}$ likewise. Finally, by the expansion (1.2), this number is equal to

$$
\begin{aligned}
& \sum_{t_{1}=1}^{n} \ldots \sum_{t_{c}=1}^{n} \sum_{j_{1}=1}^{n} \ldots \sum_{j_{d}=1}^{n} A\left(n ; t_{1}, \ldots, t_{c} ; j_{1}, \ldots, j_{d}\right) \\
& \quad \times(-1)^{t_{1}+\cdots+t_{c}+s_{1}+\cdots+s_{c}} \prod_{l=1}^{c} \Delta_{k_{l}}^{s_{c+1-l}-1}\left(\begin{array}{c}
k_{l}-c-1 \\
t_{c+1-l}-1
\end{array}\right) \\
& \quad \times\left.\prod_{l=n-d+1}^{n} \delta_{k_{l}}^{i_{l-n+d}-1}\left(\begin{array}{c}
k_{l}-n+d-2+j_{l-n+d} \\
j_{l-n+d}-1
\end{array}\right)\right|_{\left(k_{1}, \ldots, k_{c}, k_{n-d+1}, \ldots, k_{n}\right)=\left((c+1)^{c},(n-d)^{d}\right)}
\end{aligned}
$$

Since $\Delta_{x}\left(\begin{array}{l}x \\ n\end{array}\right)=\left(\begin{array}{c}x \\ n-1\end{array}\right)$ and $\delta_{x}\left(\begin{array}{l}x \\ n\end{array}\right)=\left(\begin{array}{l}x-1 \\ n-1\end{array}\right)$, this is equal to

$$
\begin{aligned}
\sum_{t_{1}=1}^{n} & \ldots \sum_{t_{c}=1}^{n} \sum_{j_{1}=1}^{n} \ldots \sum_{j_{d}=1}^{n} A\left(n ; t_{1}, \ldots, t_{c} ; j_{1}, \ldots, j_{d}\right) \\
& \times(-1)^{t_{1}+\cdots+t_{c}+s_{1}+\cdots+s_{c}} \prod_{l=1}^{c}\left(\begin{array}{c}
k_{l}-c-1 \\
t_{c+1-l}-s_{c+1-l}
\end{array}\right) \\
& \times\left.\prod_{l=n-d+1}^{n}\left(\begin{array}{c}
k_{l}-n+d-1+j_{l-n+d}-i_{l-n+d} \\
j_{l-n+d}-i_{l-n+d}
\end{array}\right)\right|_{\left(k_{1}, \ldots, k_{c}, k_{n-d+1}, \ldots, k_{n}\right)=\left((c+1)^{c},(n-d)^{d}\right)} \\
= & \sum_{t_{1}=1}^{n} \ldots \sum_{t_{c}=1}^{n} \sum_{j_{1}=1}^{n} \ldots \sum_{j_{d}=1}^{n} A\left(n ; t_{1}, \ldots, t_{c} ; j_{1}, \ldots, j_{d}\right)(-1)^{t_{1}+\cdots+t_{c}+s_{1}+\cdots+s_{c}} \\
& \times \prod_{l=1}^{c}\left(\begin{array}{c}
0 \\
t_{c+1-l}-s_{c+1-l}
\end{array}\right) \prod_{l=n-d+1}^{n}\left(\begin{array}{c}
j_{l-n+d}-i_{l-n+d}-1 \\
j_{l-n+d}-i_{l-n+d}
\end{array}\right) .
\end{aligned}
$$

As $\left(\begin{array}{l}0 \\ k\end{array}\right)=\delta_{0, k}=\left(\begin{array}{c}k-1 \\ k\end{array}\right)$ for all integers $k$, this simplifies to $A\left(n ; s_{1}, \ldots, s_{c} ; i_{1}, \ldots, i_{d}\right)$ and concludes the proof of Theorem 1. 
5 A useful relation between the numbers $A\left(n ; s_{1}, \ldots, s_{c} ; i_{1}, \ldots, i_{d}\right)$ with constant $c+d$ and a formula for $A_{n, i, j}$

In order to prove (1.3) we make use of the identity

$$
\alpha\left(n ; k_{1}, \ldots, k_{n}\right)=(-1)^{n-1} \alpha\left(n ; k_{2}, \ldots, k_{n}, k_{1}-n\right),
$$

which can be found in [3, Lemma 5]. It implies

$$
\alpha\left(n ; k_{1}, \ldots, k_{n}\right)=(-1)^{t n-t} \alpha\left(n ; k_{t+1}, \ldots, k_{n}, k_{1}-n, k_{2}-n, \ldots, k_{t}-n\right)
$$

for $t \geq 0$. Moreover, for every integer $z$,

$$
\alpha\left(n ; k_{1}, \ldots, k_{n}\right)=\alpha\left(n ; k_{1}+z, \ldots, k_{n}+z\right) .
$$

Therefore,

$$
\begin{aligned}
& \sum_{s_{1}=1}^{n} \ldots \sum_{s_{c}=1}^{n} \sum_{i_{1}=1}^{n} \cdots \sum_{i_{d}=1}^{n} A\left(n ; s_{1}, \ldots, s_{c} ; i_{1}, \ldots, i_{d}\right) \\
& \times(-1)^{s_{1}+\cdots+s_{c}+c} \prod_{l=1}^{c}\left(\begin{array}{c}
k_{l}-c-1 \\
s_{c+1-l}-1
\end{array}\right) \prod_{l=n-d+1}^{n}\left(\begin{array}{c}
k_{l}-n+d-2+i_{l-n+d} \\
i_{l-n+d}-1
\end{array}\right) \\
& =\alpha\left(n ; k_{1}, \ldots, k_{c}, c+1, c+2, \ldots, n-d, k_{n-d+1}, \ldots, k_{n}\right) \\
& =(-1)^{t n-t} \alpha\left(n ; k_{t+1}, \ldots, k_{c}, c+1, c+2, \ldots, n-d,\right. \\
& \left.k_{n-d+1}, \ldots, k_{n}, k_{1}-n, k_{2}-n, \ldots, k_{t}-n\right) \\
& =(-1)^{t n-t} \alpha\left(n ; k_{t+1}-t, \ldots, k_{c}-t, c+1-t, c+2-t, \ldots\right. \text {, } \\
& \left.n-d-t, k_{n-d+1}-t, \ldots, k_{n}-t, k_{1}-n-t, \ldots, k_{t}-n-t\right) \\
& =\sum_{s_{1}=1}^{n} \ldots \sum_{s_{c-t}=1}^{n} \sum_{i_{1}=1}^{n} \ldots \sum_{i_{d+t}=1}^{n} A\left(n ; s_{1}, \ldots, s_{c-t} ; i_{1}, \ldots, i_{d+t}\right) \\
& \times(-1)^{s_{1}+\cdots+s_{c-t}+c+t n}\left(\begin{array}{c}
k_{t+1}-c-1 \\
s_{c-t}-1
\end{array}\right)\left(\begin{array}{c}
k_{t+2}-c-1 \\
s_{c-t-1}-1
\end{array}\right) \cdots\left(\begin{array}{c}
k_{c}-c-1 \\
s_{1}-1
\end{array}\right) \\
& \times\left(\begin{array}{c}
k_{n-d+1}-n+d-2+i_{1} \\
i_{1}-1
\end{array}\right) \ldots\left(\begin{array}{c}
k_{n}-n+d-2+i_{d} \\
i_{d}-1
\end{array}\right) \\
& \times\left(\begin{array}{c}
k_{1}-2 n+d-2+i_{d+1} \\
i_{d+1}-1
\end{array}\right) \\
& \times\left(\begin{array}{c}
k_{2}-2 n+d-2+i_{d+2} \\
i_{d+2}-1
\end{array}\right) \cdots\left(\begin{array}{c}
k_{t}-2 n+d-2+i_{d+t} \\
i_{d+t}-1
\end{array}\right) .
\end{aligned}
$$


This implies that

$$
\begin{aligned}
& \sum_{s_{c-t+1}=1}^{n} \sum_{s_{c-t+2}=1}^{n} \ldots \sum_{s_{c}=1}^{n} A\left(n ; s_{1}, \ldots, s_{c} ; i_{1}, \ldots, i_{d}\right) \\
& \quad \times(-1)^{s_{c-t+1}+\cdots+s_{c}}\left(\begin{array}{c}
k_{1}-c-1 \\
s_{c}-1
\end{array}\right) \ldots\left(\begin{array}{c}
k_{t}-c-1 \\
s_{c-t+1}-1
\end{array}\right) \\
& =\sum_{i_{d+1}=1}^{n} \sum_{i_{d+2}=1}^{n} \ldots \sum_{i_{d+t}=1}^{n} A\left(n ; s_{1}, \ldots, s_{c-t} ; i_{1}, \ldots, i_{d+t}\right) \\
& \quad \times(-1)^{t n}\left(\begin{array}{c}
k_{1}-2 n+d-2+i_{d+1} \\
i_{d+1}-1
\end{array}\right) \ldots\left(\begin{array}{c}
k_{t}-2 n+d-2+i_{d+t} \\
i_{d+t}-1
\end{array}\right) .
\end{aligned}
$$

By the Chu-Vandermonde summation, the right-hand side is equal to

$$
\begin{aligned}
& \sum_{i_{d+1}=1}^{n} \sum_{i_{d+2}=1}^{n} \ldots \sum_{i_{d+t}=1}^{n} \sum_{s_{c}=1}^{i_{d+1}} \sum_{s_{c-1}=1}^{i_{d+2}} \ldots \sum_{s_{c-t+1}=1}^{i_{d+t}} A\left(n ; s_{1}, \ldots, s_{c-t} ; i_{1}, \ldots, i_{d+t}\right) \\
& \times(-1)^{t n}\left(\begin{array}{c}
k_{1}-c-1 \\
s_{c}-1
\end{array}\right)\left(\begin{array}{c}
-2 n+d-1+i_{d+1}+c \\
i_{d+1}-s_{c}
\end{array}\right)\left(\begin{array}{c}
k_{2}-c-1 \\
s_{c-1}-1
\end{array}\right) \\
& \quad \times\left(\begin{array}{c}
-2 n+d-1+i_{d+2}+c \\
i_{d+2}-s_{c-1}
\end{array}\right) \ldots\left(\begin{array}{c}
k_{t}-c-1 \\
s_{c-t+1}-1
\end{array}\right)\left(\begin{array}{c}
-2 n+d-1+i_{d+t}+c \\
i_{d+t}-s_{c-t+1}
\end{array}\right) .
\end{aligned}
$$

Consequently,

$$
\begin{aligned}
& A\left(n ; s_{1}, \ldots, s_{c} ; i_{1}, \ldots, i_{d}\right) \\
& =\sum_{i_{d+1}=s_{c}}^{n} \sum_{i_{d+2}=s_{c-1}}^{n} \ldots \sum_{i_{d+t}=s_{c-t+1}}^{n} A\left(n ; s_{1}, \ldots, s_{c-t} ; i_{1}, \ldots, i_{d+t}\right) \\
& \quad \times(-1)^{s_{c-t+1}+\cdots+s_{c}+t n}\left(\begin{array}{c}
-2 n+d-1+i_{d+1}+c \\
i_{d+1}-s_{c}
\end{array}\right) \\
& \quad \times \cdots \times\left(\begin{array}{c}
-2 n+d-1+i_{d+t}+c \\
i_{d+t}-s_{c-t+1}
\end{array}\right) .
\end{aligned}
$$

Equivalently, by using $\left(\begin{array}{l}n \\ k\end{array}\right)=(-1)^{k}\left(\begin{array}{c}k-n-1 \\ k\end{array}\right)$,

$$
\begin{aligned}
& A\left(n ; s_{1}, \ldots, s_{c} ; i_{1}, \ldots, i_{d}\right) \\
& \quad=\sum_{i_{d+1}=s_{c}}^{n} \sum_{i_{d+2}=s_{c-1}}^{n} \ldots \sum_{i_{d+t}=s_{c-t+1}}^{n} A\left(n ; s_{1}, \ldots, s_{c-t} ; i_{1}, \ldots, i_{d+t}\right) \\
& \quad \times(-1)^{i_{d+1}+\cdots+i_{d+t}+t n}\left(\begin{array}{c}
2 n-c-d-s_{c} \\
i_{d+1}-s_{c}
\end{array}\right) \ldots\left(\begin{array}{c}
2 n-c-d-s_{c-t+1} \\
i_{d+t}-s_{c-t+1}
\end{array}\right) .
\end{aligned}
$$


Likewise, it is possible to show that

$$
\begin{aligned}
A\left(n ; s_{1}, \ldots, s_{c} ; i_{1}, \ldots, i_{d}\right) & \\
= & \sum_{s_{c+1}=i_{d}}^{n} \sum_{s_{c+2}=i_{d-1}}^{n} \ldots \sum_{s_{c+t}=i_{d-t+1}}^{n} A\left(n ; s_{1}, \ldots, s_{c+t} ; i_{1}, \ldots, i_{d-t}\right) \\
& \quad \times(-1)^{s_{c+1}+\cdots+s_{c+t}+t n}\left(\begin{array}{c}
2 n-c-d-i_{d} \\
s_{c+1}-i_{d}
\end{array}\right) \ldots\left(\begin{array}{c}
2 n-c-d-i_{d-t+1} \\
s_{c+t}-i_{d-t+1}
\end{array}\right) .
\end{aligned}
$$

The significance of these identities is that they show that the numbers $A\left(n ; s_{1}, \ldots, s_{c}\right.$; $i_{1}, \ldots, i_{d}$ ) with constant $c+d$ are linearly related.

In the special case $t=1, c=2$ and $d=0$, this gives

$$
A\left(n ; s_{1}, s_{2} ;-\right)=\sum_{i_{1}=s_{2}}^{n}(-1)^{n+i_{1}}\left(\begin{array}{c}
2 n-2-s_{2} \\
i_{1}-s_{2}
\end{array}\right) A\left(n ; s_{1} ; i_{1}\right) .
$$

We fix the following notation: $A_{n}:=A(n ;-,-), A_{n, i}:=A(n ; i ;-), B_{n, i, j}=$ $A(n ; i ; j)$ and $A_{n, i, j}=A(n ; i, j ;-)$. That is, $A_{n}$ is the total number of $n \times n$ alternating sign matrices, $A_{n, i}$ is the number of $n \times n$ alternating sign matrices where the unique 1 in the bottom (equivalently top) row is in column $i, B_{n, i, j}$ is the number of $n \times n$ alternating sign matrices where the unique 1 in the top row is in column $j$ and the unique 1 in the bottom row is in column $i$ and, finally, $A_{n, i, j}$ is the number of monotone triangles with bottom row $(1,2, \ldots, i-1, i+1, \ldots, j-1, j+1, \ldots, n)$. (Or, equivalently, $A_{n, i, j}$ is the number of monotone $(2, n)$-trapezoids with top row $(i, j)$ and bottom row $(1,2, \ldots, n)$. In the introduction, an interpretation of $A_{n, i, j}$ in terms of alternating sign matrices is provided.)

Stroganov [8, Formula (34)] has shown that

$$
\begin{aligned}
B_{n, i, j}-B_{n, i-1, j-1}= & \frac{1}{A_{n-1}}\left(A_{n-1, i-1}\left(A_{n, j}-A_{n, j-1}\right)\right. \\
& \left.+A_{n-1, j-1}\left(A_{n, i}-A_{n, i-1}\right)\right) .
\end{aligned}
$$

(See [2] for generalisations of this result.) By the combinatorial interpretation of the numbers $B_{n, i, j}$ we have $B_{n, 1, j}=A_{n-1, j-1}$ and thus

$$
\begin{aligned}
B_{n, i, k}= & A_{n-1, k-i}+\sum_{l=2}^{i}\left(B_{n, l, k-i+l}-B_{n, l-1, k-i+l-1}\right) \\
= & A_{n-1, k-i}+\frac{1}{A_{n-1}} \sum_{l=2}^{i}\left(A_{n-1, l-1}\left(A_{n, k-i+l}-A_{n, k-i+l-1}\right)\right. \\
& \left.+A_{n-1, k-i+l-1}\left(A_{n, l}-A_{n, l-1}\right)\right) .
\end{aligned}
$$


If we define $A_{n, l}=0$ for $l \notin\{1,2, \ldots, n\}$ then

$$
\begin{aligned}
B_{n, i, k}= & \frac{1}{A_{n-1}} \sum_{l=1}^{i}\left(A_{n-1, l-1}\left(A_{n, k-i+l}-A_{n, k-i+l-1}\right)\right. \\
& \left.+A_{n-1, k-i+l-1}\left(A_{n, l}-A_{n, l-1}\right)\right) .
\end{aligned}
$$

On the other hand, by (5.2), we have

$$
A_{n, i, j}=\sum_{k=j}^{n}(-1)^{n+k}\left(\begin{array}{c}
2 n-2-j \\
k-j
\end{array}\right) B_{n, i, k} .
$$

This finally implies (1.3).

\section{Remarks}

In this paper we have shown that the number $A\left(n ; s_{1}, \ldots, s_{c} ; i_{1}, \ldots, i_{d}\right)$ of monotone $(d, n-c)$-trapezoids with prescribed top and bottom row (or, equivalently, the number of certain $(n-c-d, n)$-partial alternating sign matrices) appears as a coefficient of a specialisation of $\alpha\left(n ; k_{1}, \ldots, k_{n}\right)$ with respect to a certain (binomial) polynomial basis. We have used this to provide a formula for $A\left(n ; s_{1}, s_{2} ;-\right)$. Formulas for $A\left(n ; s_{1} ;-\right)$ and $A\left(n ; s_{1} ; i_{1}\right)$ were previously known. This raises the question of whether there exist similar formulas for the numbers $A\left(n ; s_{1}, \ldots, s_{c} ; i_{1}, \ldots, i_{d}\right)$ if $c+d \geq 3$.

Formula (5.1) shows that it suffices to restrict our attention to the coefficients

$$
A\left(n ;-, i_{1}, \ldots, i_{d}\right)
$$

Next we (re-)derive a system of linear equations for these numbers. (This computation has already appeared in [5].) To this end we need a further identity for $\alpha\left(n ; k_{1}, \ldots, k_{n}\right)$ : observe that there is a one-to-one correspondence between the number of monotone triangles with bottom row $\left(k_{1}, \ldots, k_{n}\right)$ and the number of monotone triangles with bottom row $\left(-k_{n},-k_{n-1}, \ldots,-k_{1}\right)$ and therefore

$$
\alpha\left(n ; k_{1}, \ldots, k_{n}\right)=\alpha\left(n ;-k_{n},-k_{n-1}, \ldots,-k_{1}\right) .
$$

Consequently,

$$
\begin{aligned}
& \alpha\left(n ; 1,2, \ldots, n-d, k_{n-d+1}, k_{n-d+2}, \ldots, k_{n}\right) \\
& =\alpha\left(n ;-k_{n},-k_{n-1}, \ldots,-k_{n-d+1},-n+d,-n+d+1, \ldots,-1\right) \\
& =(-1)^{d n-d} \alpha\left(n ;-n+d,-n+d+1, \ldots,-1,-k_{n}-n,-k_{n-1}-n, \ldots,\right. \\
& \left.\quad-k_{n-d+1}-n\right) \\
& =(-1)^{d n-d} \alpha\left(n ; 1,2, \ldots, n-d,-k_{n}-d+1,-k_{n-1}-d+1, \ldots,\right. \\
& \left.\quad-k_{n-d+1}-d+1\right) .
\end{aligned}
$$


In terms of the expansion (1.2), this means that

$$
\begin{aligned}
& \sum_{i_{1}=1}^{n} \ldots \sum_{i_{d}=1}^{n} A\left(n ;-; i_{1}, \ldots, i_{d}\right) \prod_{l=1}^{d}\left(\begin{array}{c}
k_{n-d+l}-n+d-2+i_{l} \\
i_{l}-1
\end{array}\right) \\
& \quad=(-1)^{d n-d} \sum_{j_{1}=1}^{n} \ldots \sum_{j_{d}=1}^{n} A\left(n ;-; j_{1}, \ldots, j_{d}\right) \prod_{l=1}^{d}\left(\begin{array}{c}
-k_{n-d+l}-n-1+j_{d+1-l} \\
j_{d+1-l}-1
\end{array}\right) .
\end{aligned}
$$

By the Chu-Vandermonde summation, we have

$$
\begin{aligned}
& \left(\begin{array}{c}
-k_{n-d+l}-n-1+j_{d+1-l} \\
j_{d+1-l}-1
\end{array}\right) \\
& =\sum_{i_{l}=1}^{j_{d+1-l}}\left(\begin{array}{c}
k_{n-d+l}-n+d-2+i_{l} \\
i_{l}-1
\end{array}\right)(-1)^{i_{l}-1}\left(\begin{array}{c}
-2 n-1+j_{d+1-l}+d \\
j_{d+1-l}-i_{l}
\end{array}\right)
\end{aligned}
$$

and, consequently,

$$
\begin{aligned}
A\left(n ;-; i_{1}, \ldots, i_{d}\right) & \\
= & \sum_{j_{1}=i_{d}}^{n} \ldots \sum_{j_{d}=i_{1}}^{n}(-1)^{d n+i_{1}+\cdots+i_{d}} A\left(n ;-; j_{1}, \ldots, j_{d}\right) \\
& \times \prod_{l=1}^{d}\left(\begin{array}{c}
-2 n-1+j_{d+1-l}+d \\
j_{d+1-l}-i_{l}
\end{array}\right) .
\end{aligned}
$$

Equivalently, by using $\left(\begin{array}{l}n \\ k\end{array}\right)=(-1)^{k}\left(\begin{array}{c}k-n-1 \\ k\end{array}\right)$,

$$
\begin{aligned}
& A\left(n ;-; i_{1}, \ldots, i_{d}\right) \\
& \quad=\sum_{j_{1}=i_{1}}^{n} \ldots \sum_{j_{d}=i_{d}}^{n}(-1)^{d n+j_{1}+\cdots+j_{d}} A\left(n ;-; j_{d}, \ldots, j_{1}\right) \prod_{l=1}^{d}\left(\begin{array}{c}
2 n-i_{l}-d \\
j_{l}-i_{l}
\end{array}\right),
\end{aligned}
$$

where $1 \leq i_{1}, i_{2}, \ldots, i_{d} \leq n$. (This is Conjecture 5 from [5].)

Remark 1 In fact, we have used the principle that certain identities for $\alpha\left(n ; k_{1}, \ldots, k_{n}\right)$ translate to identities for the coefficients $A\left(n ; s_{1}, \ldots, s_{c} ; i_{1}, \ldots, i_{d}\right)$. Another (much less interesting) example in this respect is

$$
\begin{aligned}
\alpha\left(n ; k_{1}, \ldots, k_{c}, c+1, \ldots, n-d, k_{n-d+1}, \ldots, k_{n}\right) \\
=\alpha\left(n ;-k_{n},-k_{n-1}, \ldots,-k_{n-d+1},-n+d, \ldots,-c-1,-k_{c}, \ldots,-k_{1}\right) \\
=\alpha\left(n ; n+1-k_{n}, n+1-k_{n-1}, \ldots,\right. \\
\left.\quad n+1-k_{n-d+1}, d+1, \ldots, n-c, n+1-k_{c}, \ldots, n+1-k_{1}\right),
\end{aligned}
$$


which translates in a similar way to

$$
A\left(n ; s_{1}, \ldots, s_{c} ; i_{1}, \ldots, i_{d}\right)=A\left(n ; i_{1}, \ldots, i_{d} ; s_{1}, \ldots, s_{c}\right),
$$

an identity that is of course (almost) obvious from the combinatorial interpretation if $s_{1}<s_{2}<\cdots<s_{c}$ and $i_{1}<i_{2}<\cdots<i_{d}$.

For fixed $n$, the system of linear equations (6.1) provides a total of $n^{d}$ linear equations for the $n^{d}$ numbers $A\left(n ;-; i_{1}, \ldots, i_{d}\right), 1 \leq i_{1}, i_{2}, \ldots, i_{d} \leq n$. However, computer experiments show that there is a certain dependency among these linear equations.

For the case $d=1$ (i.e. the numbers $A(n ;-; i)=A_{n, i}$ of the refined alternating sign matrix theorem), it was shown in [3] that (6.1) together with $A_{n, 1}=A_{n-1}$ and the symmetry $A_{n, i}=A_{n, n+1-i}$ for $1 \leq i \leq n$ (which easily follows from the combinatorial interpretation of the numbers $A_{n, i}$ ) provides a system of linear equations that determines the numbers $A_{n, i}$ uniquely. In [5] we have conjectured that this extends to the case $d=2$, i.e. the numbers $A(n ;-; i, j)=A_{n, i, j}$. In this case, we have to add $A_{n, i, n}=A_{n-1, i}$, the near-symmetry

$$
A_{n, i, j}=A_{n, n+1-j, n+1-i}
$$

for all $i, j \in\{1,2, \ldots, n\}$ unless $(i, j) \notin\{(n-1,1),(n, 2)\}$ and $A_{n, n-1,1}-A_{n-1}=$ $A_{n, n, 2}$. If $i<j$ this near-symmetry also easily follows from the combinatorial interpretation of the numbers $A_{n, i, j}$, however, for $i \geq j$, we took some efforts in [5] to derive it. It would be of interest to see whether this (conjectural) behaviour extends to the case $d>2$. By Cramer's rule this would then at least provide a determinantal expression for the numbers $A\left(n ;-, i_{1}, \ldots, i_{d}\right)$.

As mentioned above, monotone $(d, n-c)$-trapezoids correspond to certain $(n-c-d, n)$-partial alternating sign matrices. The latter are generalisations of alternating sign matrices with loosened column-sum restrictions. Clearly, a next natural step would be to consider even more general partial alternating sign matrices, where we loosen the column-sum restrictions and the row-sum restrictions. In particular, the quadruply refined enumeration of alternating sign matrices where we fix top and bottom row as well as the first and the last column of the alternating sign matrix, would be of major interest.

\section{References}

1. Bressoud, D.M.: Proofs and Confirmations, the Story of the Alternating Sign Matrix Conjecture. Cambridge University Press, Cambridge (1999)

2. Colomo, F., Pranko, A.G.: On two-point boundary correlations in the six-vertex model with DWBC. J. Stat. Mech. 05010 (2005)

3. Fischer, I.: A new proof of the refined alternating sign matrix theorem. J. Comb. Theory: Ser. A 114, 253-264 (2007)

4. Fischer, I.: The operator formula for monotone triangles-simplified proof and three generalizations. J. Comb. Theory: Ser. A (in press). arXiv:0903.4628

5. Fischer, I., Romik, D.: More refined enumerations of alternating sign matrices. Adv. Math. 222, 20042035 (2009) 
6. Karklinsky, M., Romik, D.: A formula for the doubly refined enumeration of alternating sign matrices. Adv. Appl. Math. 45, 28-35 (2010)

7. Mills, W.H., Robbins, D.P., Rumsey, H.: Alternating sign matrices and descending plane partitions. J. Comb. Theory: Ser. A 34, 340-359 (1983)

8. Stroganov, Yu.G.: A new way to deal with Izergin-Korepin determinant at root of unity. arXiv: math-ph/0204042

9. Zeilberger, D.: Proof of the alternating sign matrix conjecture. Electron. J. Comb. 3, R13 (1996) 